\title{
NANORIBBED TORSIONAL OSCILLATORS OPTIMIZED FOR HIGH-FREQUENCY FORCE SENSING
}

\author{
Benjamin W. Chui, Charles T. Rettner, H. Jonathon Mamin, Raffi Budakian, and Daniel Rugar \\ IBM Research Division, Almaden Research Center, San Jose, CA 95120 \\ ben.chui@california.com, rugar@almaden.ibm.com
}

\begin{abstract}
In this paper, we focus on ultrasensitive cantilevers for the detection of nuclear magnetic resonance (NMR) at frequencies that coincide with the nuclear spin precession (Larmor) frequency, around $1 \mathrm{MHz}$. The small size of these cantilevers precludes the usual practice of locating the reflective paddle near the tip, so a torsional configuration has to be used to allow the paddle to be situated at the distal end, providing improved optical access. These torsional oscillators, fabricated in part with electron-beam lithography, have ribs $200 \mathrm{~nm}$ wide and $1.5 \mu \mathrm{m}$ tall attached to arms $200 \mathrm{~nm}$ thick and $12 \mu \mathrm{m}$ long. Fabrication details and characterization results are presented.
\end{abstract}

\section{INTRODUCTION}

Previously, we have reported on mass-loaded cantilevers for magnetic resonance force microscopy (MRFM) [1]. These divingboard style cantilevers, which were $90 \mu \mathrm{m}$ long with a natural frequency around $5 \mathrm{kHz}$, have been used to detect electron spin resonance with close to single-spin sensitivity. In that experiment, the cantilevers measured the low-frequency modulation of the longitudinal component of the electron spin [2]. Ultimately, we hope to extend this capability to nuclear-spin detection and explore a new mode of operation where the cantilever oscillates synchronously with the transverse component of the spin precession [3]. In the case of hydrogen nuclei (i.e., protons) in a 250-Gauss magnetic field, this precession frequency (called the Larmor frequency) is approximately $1 \mathrm{MHz}$.

In order to operate at megahertz frequencies while maintaining attonewton force sensitivity, the cantilever must be thin (e.g., 300 $\mathrm{nm}$ thick) and short (e.g., $20 \mu \mathrm{m}$ or less) if the standard divingboard configuration is to be used. Sensing cantilever motion with our standard technique of optical interferometry in this case, however, could be challenging. Since the cantilever is positioned

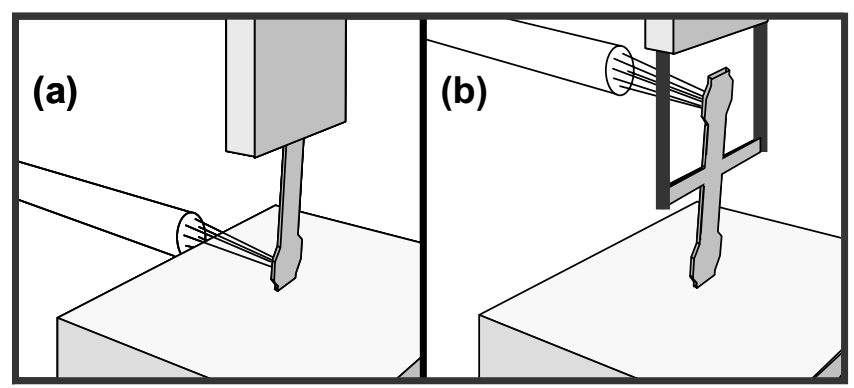

Figure 1: (a) Conventional and (b) torsional cantilevers used in scanning. In (a), if the cantilever is small and the paddle is located close to the tip, the optical path from the laser interferometer to the paddle can be blocked by the sample. Using a torsional cantilever as shown in (b) can improve optical access. very near (and in our case perpendicular to) the sample, the sample can block optical access to the tip where the reflective paddle is located (Fig. 1a). If the paddle is moved further back (away from the tip and towards the base), a mechanical disadvantage would be introduced with respect to tip motion, ultimately reducing optical detection sensitivity. Ideally, one would like to spatially separate the optically sensed region from the probe-tip region without introducing a mechanical disadvantage or sacrificing high-frequency operation (Fig. 1b).

\section{DEVICE DESIGN}

To meet these challenges, we have designed and fabricated torsional oscillators with the following characteristics:

- Resonance frequency of approximately $1 \mathrm{MHz}$, with spring constant below $1 \mathrm{~N} / \mathrm{m}$ (i.e., low-mass, small-size)

- High Q (use single-crystal silicon)

- Adequate frequency separation between the fundamental mode and spurious higher-order modes

- Optical sensing unaffected by sample proximity (i.e., place the reflective paddle on the arm that is opposite the tip)

The resulting optimized design is shown in Fig. 2. Note the small size of the device $(12 \mu \mathrm{m}$-long arms and $2 \mu \mathrm{m}$-long hinges, both $120 \mathrm{~nm}$ thick) compared to our previous ones $[\mathbf{1 , 4}$. To stiffen the unwanted flapping modes without adding excessive mass, a tall but narrow "nanorib" $(1.5 \mu \mathrm{m}$ by $200 \mathrm{~nm})$ is incorporated along the arms perpendicular to the plane of cantilever.

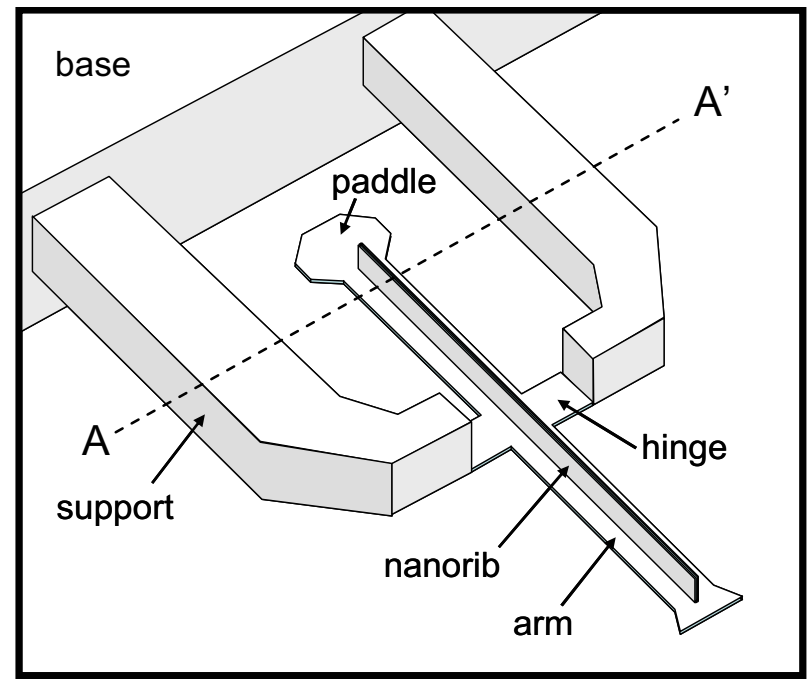

Figure 2: Nanoribbed torsional cantilever design. The arms and the hinge are all $120 \mathrm{~nm}$ thick. Each of the two arms is $12 \mu \mathrm{m}$ long and $2 \mu \mathrm{m}$ wide. The hinge on each side of the arm is $2 \mu \mathrm{m}$ long and $2 \mu \mathrm{m}$ wide. The nanorib is $200 \mathrm{~nm}$ wide and $1.5 \mu \mathrm{m}$ tall. Note that the supports are thickened for rigidity. 


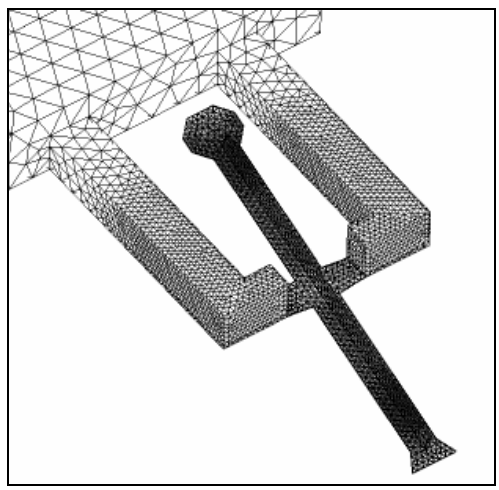

$1.20 \mathrm{MHz}$

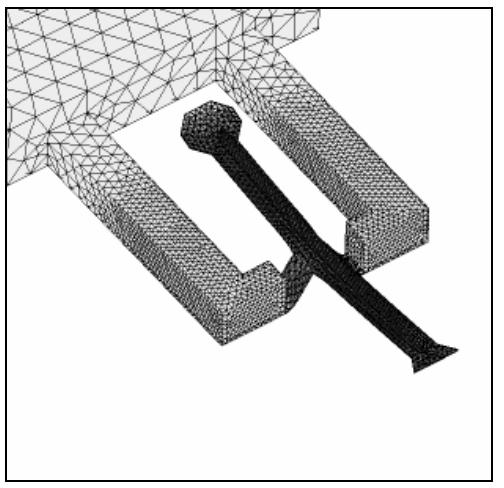

$6.31 \mathrm{MHz}$

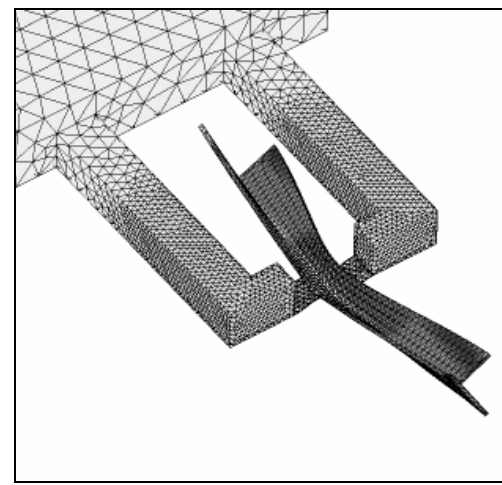

$10.0 \mathrm{MHz}$

Figure 3: The fundamental (torsional) mode of the cantilever is at $1.20 \mathrm{MHz}$ according to finite element analysis, while the next two modes are at $6.31 \mathrm{MHz}$ and $10.0 \mathrm{MHz}$ respectively, i.e. at least five times higher than the fundamental.

The fundamental, torsional mode is designed to be at $1.2 \mathrm{MHz}$, and the next mode is more than five times higher according to finite element analysis (Fig. 3). The calculated torsional stiffness is $0.6 \mathrm{~N} / \mathrm{m}$. As required, the reflector paddle is located at the opposite end to the tip, providing improved optical access.

\section{DEVICE FABRICATION}

The fabrication process is shown in Fig. 4. Note that electronbeam lithography is used to pattern the nanoribs. In fact, e-beam lithography is juxtaposed with conventional lithography and multistep plasma etching to form sub-micron, high-aspect-ratio features coupled to larger, flat structures. Also note that for our application, the cantilever tip must protrude from the edge of the chip in order to access the sample; i.e., a surface-released device [5] would not be suitable. We therefore had to adopt a bulk-release approach.

The starting material for our process is a silicon-on-insulator (SOI) wafer with a $1.5-\mu \mathrm{m}$ top silicon layer (Fig. 4a). A 200-nm thermal oxide layer is grown and patterned (Fig. 4b), and on top of that a second pattern is written with e-beam on 300-nm spin-onglass (SOG) type resist (Fig. 4c). The oxide mask defines the gross features of the device (the base and support), while the electronbeam mask defines the sub-micron rib. The wafer with the composite oxide-SOG mask is then subject to a timed plasma etch that forms the rib (Fig. 4d). Note that the etch stops just short of the SOI buried oxide. In this case a highly non-isotropic etch is desirable since it creates a uniform cross-section for the thin, narrow rib. Etch-rate uniformity across the wafer is also important, since the amount of top silicon left after the etch determines the final thickness of the arm. The oxide-SOG mask is then removed with HF.

A low-temperature-oxide (LTO) layer is grown and patterned, and is used as a mask for the ensuing selective epitaxy deposition (Fig. 4e). In this step, areas not covered by oxide (i.e., the base and most of the support) will be thickened by the growth of singlecrystal silicon epitaxy (Fig. 4f), making them more rigid and less susceptible to unwanted low-frequency modes. An additional lithographic step (not necessarily e-beam) plus one more plasma etch (stopping on the buried oxide) is used to define the arms of the

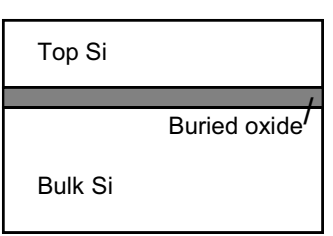

(a) Start with SOI wafer

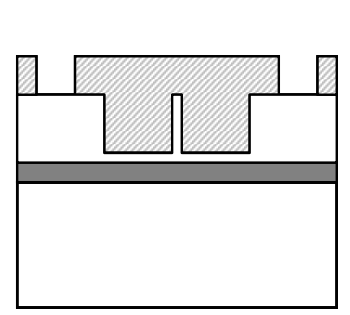

(e) Deposit and pattern LTO mask

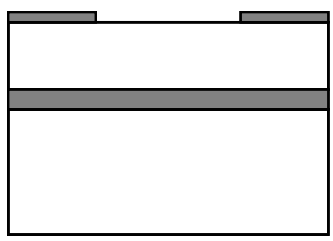

(b) Grow and pattern thin-oxide mask

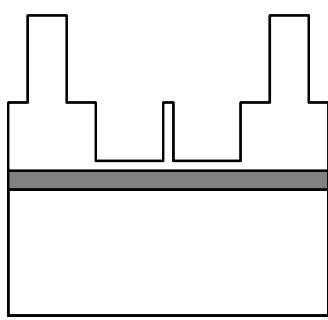

(f) Grow selective Si epi on supports; strip LTO

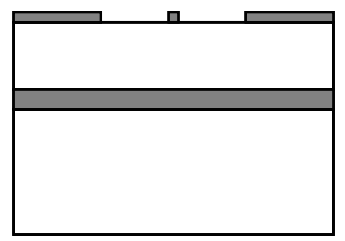

(c) Add e-beam mask

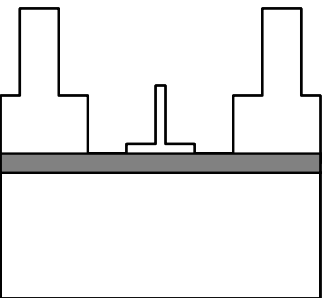

(g) Pattern and etch cantilever flange

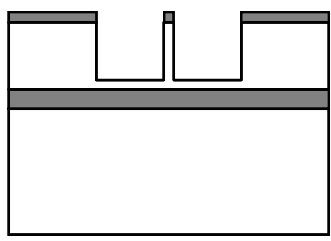

(d) Use timed plasma etch to form nanoribs

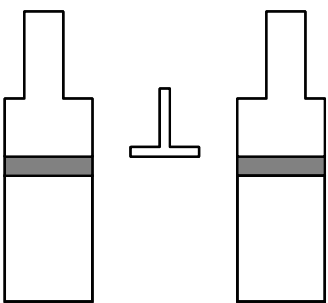

(h) Backside etch and HF release
Figure 4: Fabrication process flow for the nanoribbed torsional cantilever (viewed along section AA' of Figure 2). Note the electron-beam lithography step (c) and the subsequent timed plasma etch (d) used to define the high-aspectratio ribs. In spite of the very delicate cantilever structure, a bulk-release approach based on through-wafer backside deep reactive ion etch (DRIE) is used so that the resulting device can protrude from the edge of the chip just like a scanning probe tip. 


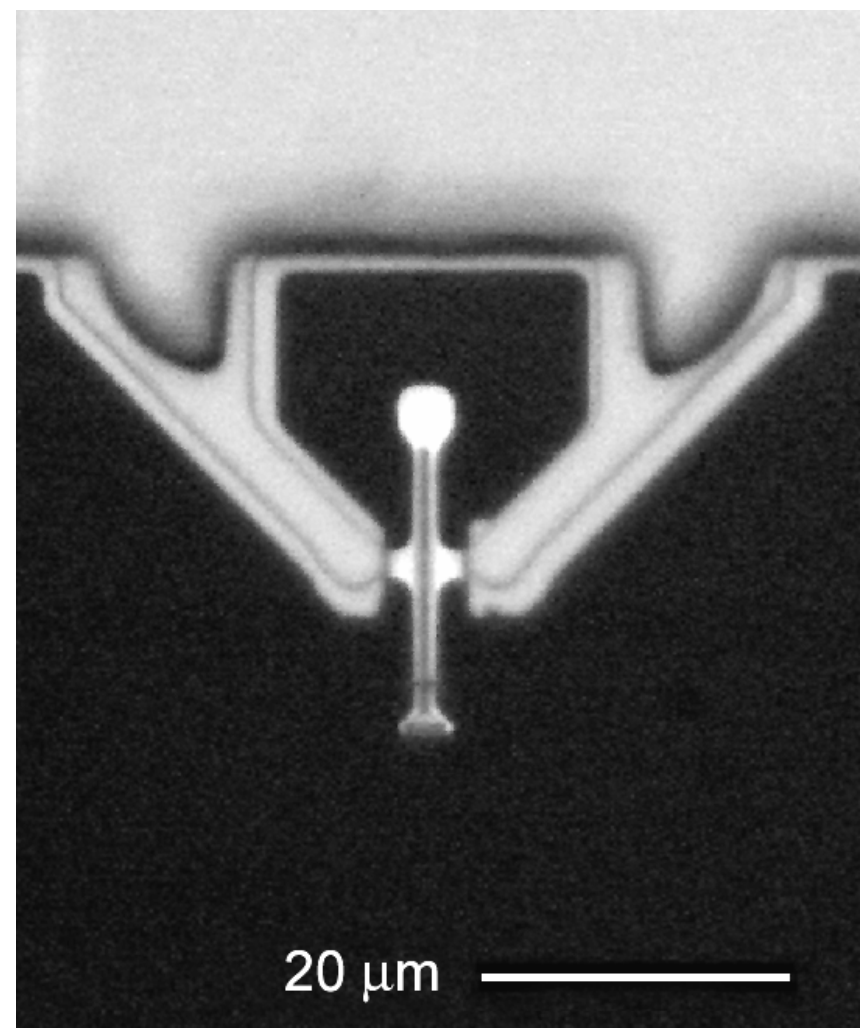

Figure 5: Optical image (top view) of a fully released torsional cantilever. Note that when used in actual scanning, the tip will be functionalized with an appropriate magnetic particle or coating.

cantilever (Fig. 4g). Finally, a nitride-LTO bi-layer is grown to protect the top side of the wafer, and a backside DRIE step (with the buried oxide as the etch-stop) is used to create through holes in the wafer. The top-side nitride is then plasma-etched away, and finally an HF vapor etch is used to remove the buried oxide and top-side LTO and release the finished devices (Fig. 4h).

Optical and SEM images of fully released, single-crystal silicon torsional oscillators are shown in Figures 5 and 6 . Figure 5 shows a standard cantilever design, while Fig. 6 shows a design variation that has an extra nanorib across the hinge for reinforcement. Both designs exhibit the same basic torsional behavior.

\section{DEVICE CHARACTERIZATION}

A standard-design torsional cantilever has been characterized in vacuum at room temperature, indicating that $f_{0}=1.42 \mathrm{MHz}$ and $Q \sim 6000$ (Fig. 7). In comparison, finite element analysis predicts $f_{0}=1.20 \mathrm{MHz}$. Primary sources of discrepancy, we believe, include lithographic alignment error and thick photoresist-related distortion, as well as deviations in cantilever hinge thickness due to etch-rate non-uniformity across the wafer. (The resonant frequency of the cantilever is highly sensitive to hinge thickness.)

Efforts are also being made to study the behavior of $f_{0}$ and $Q$ in vacuum at low temperatures (down to liquid $\mathrm{N}_{2}$ and eventually liquid $\mathrm{He}$ temperatures). One practical difficulty we encountered with the optical measurement apparatus is that the small size of the cantilever paddle, which is $4 \mu \mathrm{m}$ in diameter, requires the optical

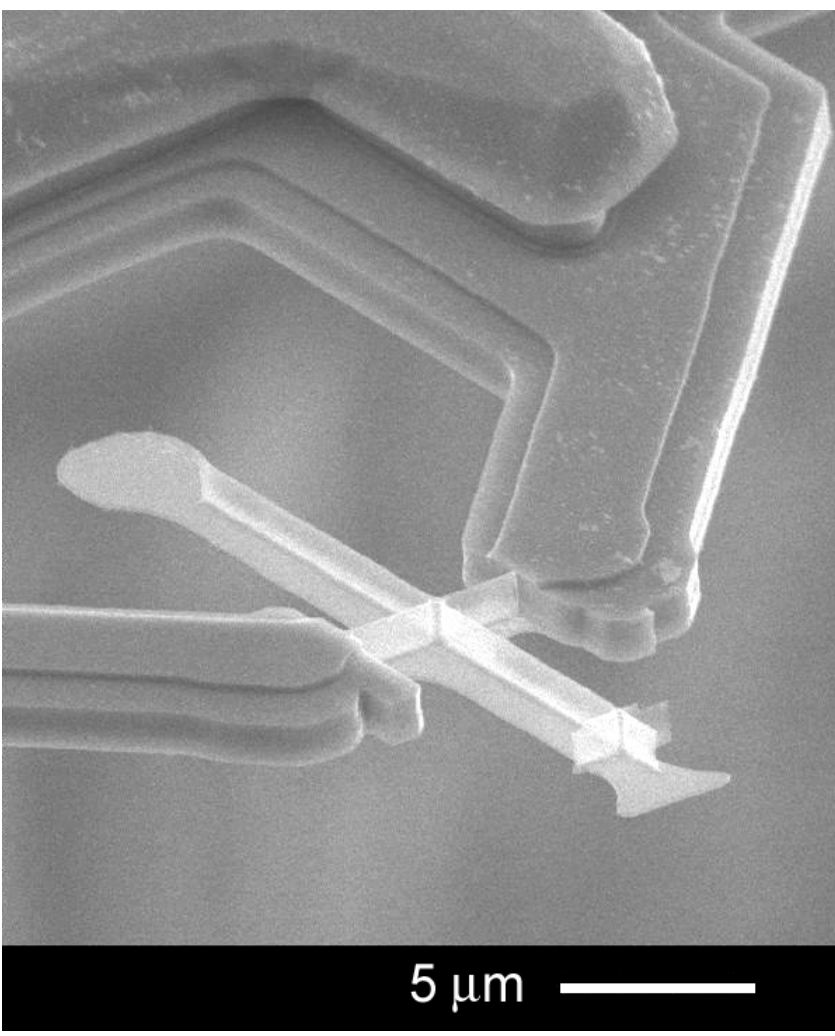

Figure 6: Scanning electron microscope image of a fully released torsional cantilever. Shown here is an alternate design with a rib on the hinge in addition to the existing one on the arm.

alignment to be extremely precise and stable at all temperatures; otherwise the laser beam would drift off the paddle causing a loss of optical signal. We have discovered that process of cooling the optical measurement apparatus does introduce a sufficiently large temperature-related mechanical shift that the laser beam becomes misaligned, and as a result we are planning to install an active beam-steering mechanism inside the vacuum chamber to combat this problem.

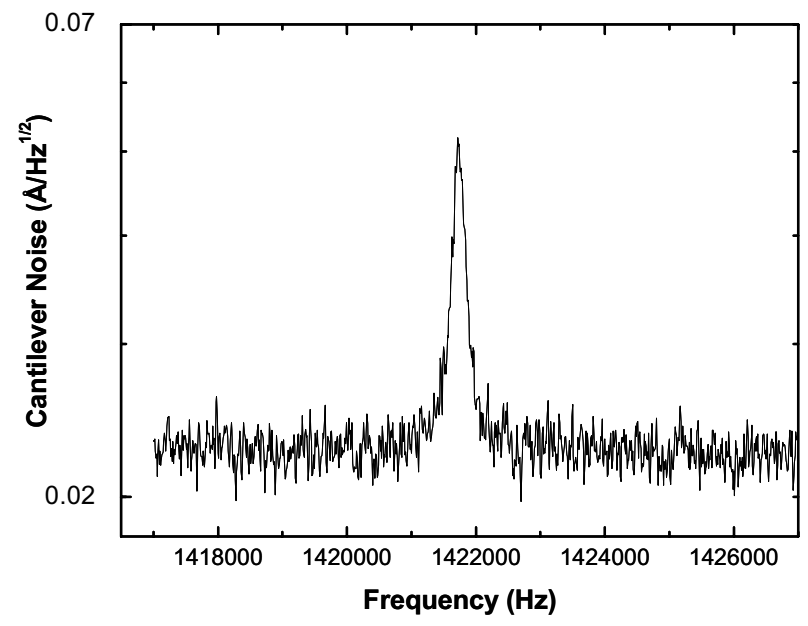

Figure 7: Frequency-domain plot of thermal noise of torsional cantilever in vacuum at room temperature, showing a fundamental frequency of $1.42 \mathrm{MHz}$ with a $Q$ of approximately 6000. 


\section{FUTURE WORK}

While the present nanoribbed cantilever largely satisfies our design and performance requirements, fabrication yields are unacceptably low due to widespread breakage during final release. Upon investigation, we have found that microtrenching tends to occur at the base of the ribs (where they meet the arm) during the rib plasma etch of Fig. 4d. This phenomenon, which is a side-effect of our highly non-isotropic plasma etch recipe, can cause cracks that extend most of the way through the thickness of the arm, eventually causing structural failure.

We have therefore completely re-designed our device to have a truss-like structure composed mostly of vertical elements, minimizing the occurrence of corners between vertical and horizontal surfaces. Prototypes of the new design have been successfully fabricated (Fig. 8). As seen in Fig. 8, the cantilever body is essentially a truss, in this case defined exclusively by electron-beam lithography. (The use of a hollow structure preserves the low-mass and high-frequency characteristics of the oscillator.) Note that the hinge is now vertically oriented as well. While reminiscent of past Hexsil devices [6], the present device is made of a different material (single-crystal silicon), is much smaller in scale and is fabricated using a substantially different process. The fabrication process largely follows that of Fig. 4, except that in step 4(d) the plasma etch now extends all the way down to the buried oxide. The one flat component of the device that remains after the plasma etchthe paddle - corresponds to a pre-fabricated mesa on the substrate. Further evaluation of the new device is in progress.

This work was supported by the DARPA MOSAIC program administered through the Army Research Office. The cantilevers were fabricated at the NSF National Nanofabrication User Network facility at Stanford University. The authors would like to thank T.W. Kenny, Y. Hishinuma, R. Shenoy, G. Yama, A. Partridge, M. Lutz, and J. McVittie for their assistance.

\section{REFERENCES}

[1] B.W. Chui, Y. Hishinuma, T.W. Kenny, H.J. Mamin, R. Budakian, D. Rugar, "Mass-loaded cantilevers with suppressed higher-order modes for magnetic resonance force microscopy," Transducers 2003 Tech. Digest, Boston, June 2003, pp. 1120-3. [2] H.J. Mamin, R. Budakian, B.W. Chui, D. Rugar, "Detection and manipulation of statistical polarization in small spin ensembles," Phys. Rev. Lett., Vol. 91, Art. 207604, Nov. 2003. [3] J.A. Sidles, J.L. Garbini, G.P. Drobny, "The theory of oscillator-coupled magnetic resonance with potential applications to molecular imaging," Rev. Sci. Instrum., Vol. 63, No. 8, pp. 3881-99, Aug. 1992.

[4] T.D. Stowe, K. Yasumura, T. Pfafman, T.W. Kenny, D. Botkin, D. Rugar, "Torsional Force Probes Optimized for Higher Order Mode Suppression," Transducers 1997 Technical Digest, Chicago, June 1997, pp. 141-4.

[5] S. Evoy, D.W. Carr, L. Sekaric, A. Olkhovets, J.M. Parpia, H.G. Craighead, "Nanofabrication and electrostatic operation of single-crystal silicon paddle oscillators," J. Appl. Phys., Vol. 86, No. 11, p. 6072-7, Dec. 1999.

[6] C.G. Keller, R.T. Howe, "Hexsil tweezers for teleoperated microassembly," Proceedings of MEMS 1997, Nagoya, Japan.
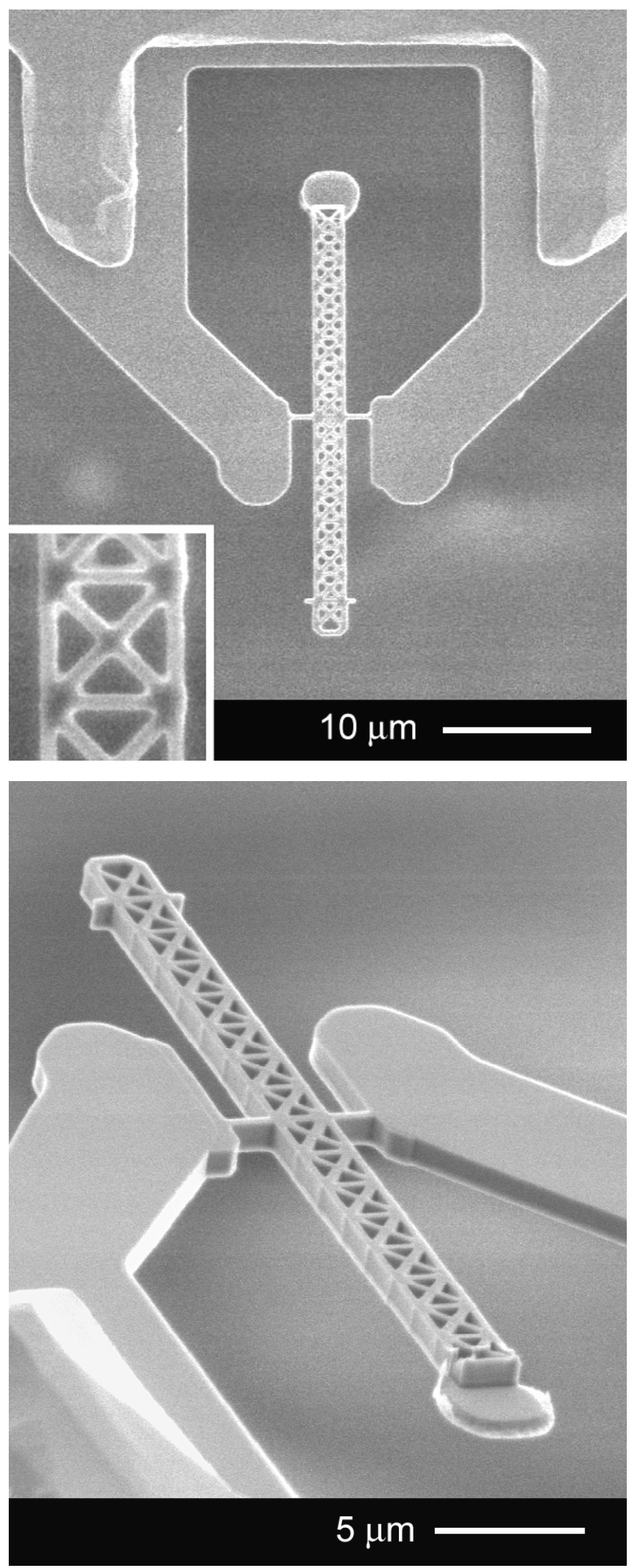

Figure 8: SEM's of fully released truss-style torsional cantilever, made of single-crystal silicon. The two arms $(16 \times 2 \times 1 \mu \mathrm{m}$ each $)$ are trusses formed by e-beam lithography and plasma etch. Note vertically oriented hinges $(2 \times 1 \times 0.25 \mu \mathrm{m}$ each $)$ and flat paddle. INSET: details of truss segment; the "unit square" is $2 \times 2 \mu \mathrm{m}$. 